\title{
28 Research Suare \\ Prevalence and factors associated with malaria among under-five children in Ethiopia: A Systematic review and Meta-analysis Protocol
}

\section{Abay Woday ( $\sim$ abaywoday@yahoo.com )}

Samara University, College of Medicine and Health Sciences, Afar Region, Ethiopia. https://orcid.org/00000003-0499-4690

\section{Mulluken Dessalegn}

Amref Health Africa

\section{Setognal Birara}

Samara University, Afar Ethiopia

Kusse Urmale

Samara University

\section{Protocol}

Keywords: prevalence, malaria, associated factors, systematic review and meta-analysis, Ethiopia

Posted Date: May 27th, 2020

DOI: https://doi.org/10.21203/rs.3.rs-28903/v1

License: @ (i) This work is licensed under a Creative Commons Attribution 4.0 International License. Read Full License 


\section{Abstract}

Background: The Sub-Saharan African countries have been carried $80 \%$ of the global burden of malaria. Consequently, malaria is still the leading cause of under-five mortality in developing nations. In Ethiopia, studies conducted regarding prevalence and associated factors of malaria among under five children are inconsistently reported and highly variable.

Objective: to determine the pooled prevalence and associated factors of malaria among under five children in Ethiopia.

Methods: The protocol for this review is registered at PROSPERO with registration number: CRD42020157886. A comprehensive search of the following electronic databases were made using: MEDLINE, EMBASE, CINAHL, Scopus, web science, HINARI, Cochrane library, Google Scholar and maternity \& infant care databases as well as grey literature uploaded at Ethiopian Health Development Journal were searched until May 2020. The quality of studies will be assessed using Joanna Briggs Institute (JBI) checklist. All identified observational studies reporting the prevalence of malaria and associated factors among under five children in Ethiopia will be considered. Three reviewers will screen all retrieved articles, conduct data extraction, and then critically appraise all identified studies. The analysis of data will be done using STATA 14.0 statistical software. We will determine the pooled prevalence and determinants of malaria among under five children using random effect model with effect size and $95 \%$ confidence interval. Heterogeneity among the included studies will be assessed through the Cochrane Q-test statistics and I2 test. Furthermore, publication bias will be checked using funnel plot and egger's test. Finally, statistical significance level will be declared at a p-value less than 0.05 .

Discussion: The result from this systematic review will inform and guide health policy planners and researchers on the burden, and determinants of under five children malaria in Ethiopia. To our knowledge, this is the first systematic review in Ethiopia. We will synthesize the findings to generate up-to-date knowledge on malaria among under five children in Ethiopia.

\section{Background}

Globally, malaria causes more than 216 million (95\% Cl: 196-263 million) cases per year. Of these cases, more than $90 \%$ are found concentrated in sub-Sahara African countries including Ethiopia [1]. Moreover, nearly half a million (429 000) deaths were caused by malaria, and more than $92 \%$ of these deaths have been happened in sub-Sahara African countries [2]. Consequently, malaria is still the leading public health problem in developing nations and it predominately affects pregnant mothers and under-five children [3]. In Ethiopia, three-fourth of the areas are malaria endemic, and more than two-thirds of its population (more than 55 million people) are at risk for malaria [4, 5]. According to the 2013 health and health related indicator report of the Ministry of Health $(\mathrm{MOH})$, malaria was one of the leading causes of morbidity and mortality in the country [6]. Several strategies, policies and malaria elimination programs had been tried at global, regional and national levels in the last decades [7-10]. Consequently, over 6.2 million malaria deaths were averted between 2000 and 2015 in subSaharan African countries [11]. However, Ethiopia was not met the 2015 target of MDG (reduce the incidence rate by $50 \%$ [12]. Thus, despite the significant decline in the burden of malaria, the disease is still one of the major public health concern in the country [13]. Furthermore, the prevalence of malaria among under five children is unacceptably high which is between $16 \%$ and $54 \%$ in the country $[4,14-17]$. 
Previous studies conducted across sub-Saharan African countries have been identified many factors associated with malaria endemic among under-five children. These includes; sex of the child, age of the child, number of bed nets available for the household members, presence of forest cover, altitude of the residence, household density (family size), living near to dam, seasonal variation and housing conditions $[4,5,15,16,18,19]$. According to the 2016 Ethiopian Demographic and Health Survey (EDHS) report, the magnitude of under-five mortality rate varies across the nine regions and the lowest in ... and the highest (125 deaths per 1000 live births) in Afar region [20]. Unfortunately, in Ethiopia, only 33\% of children under the age of five were sleeping under insecticide treated nets [21]. Consequently, the high under five mortality rate should be attributed to malaria.

In Ethiopia, limited and inconclusive studies have been conducted to date to generate information for action regarding malaria among under five children at national level. Therefore, this systematic review study attempts to estimate the pooled prevalence and factors associated with malaria among children under five in Ethiopia.

\section{Research questions}

1. What is the pooled prevalence of malaria among under five children in Ethiopia?

2. What are the major factors associated with malaria among under five children in Ethiopia?

\section{Objective}

The objectives of this systematic review and meta-analysis are; 1) to determine the pooled prevalence of malaria among under five children in Ethiopia and 2) to identify factors associated malaria among under five children in Ethiopia.

\section{Methods And Materials}

\subsection{Reporting of the review findings}

The protocol for this review is registered at PROSPERO with registration number: CRD42020157886. We will use the Preferred Reporting Items for Systematic review and Meta-analyses (PRISMA-2009) [22] (additional file 3) and (PRISMA-P 2015) [23] (Additional file 1) statements to report the findings. Moreover, we have used the guideline of the Prospero for registration.

\subsection{Study design}

A systematic review and meta-analysis will be conducted to determine the pooled prevalence of birth asphyxia and its determinants among newborns in Ethiopia.

\subsection{Eligibility Criteria}




\begin{tabular}{|c|c|c|c|c|c|c|}
\hline & $\begin{array}{l}\text { Participants } \\
\text { (P) }\end{array}$ & $\begin{array}{l}\text { Intervention } \\
\text { (I) }\end{array}$ & $\begin{array}{l}\text { comparison } \\
\text { (C) }\end{array}$ & Outcomes (O) & Study type & Limits \\
\hline $\begin{array}{l}\text { on } \\
\text { la }\end{array}$ & $\begin{array}{l}\text { Under-five } \\
\text { children or } \\
\text { children 0- } \\
59 \text { months } \\
\text { old }\end{array}$ & $\begin{array}{ll}\cdot & \text { Risk } \\
& \text { Factors } \\
& \text { OR } \\
\text {. } & \text { Causes } \\
& \text { OR } \\
\cdot & \\
& \text { Predictors } \\
& \text { OR } \\
\cdot & \\
& \text { Determina } \\
\text { nts }\end{array}$ & $\begin{array}{l}\text {-Children } \\
\text { slept under } \\
\text { ITN versus } \\
\text { not use ITN } \\
\text { - rural Vs } \\
\text { urban } \\
\text {-nutritional } \\
\text { status } \\
\text { - male Vs } \\
\text { female } \\
\text {-education } \\
\text { level of } \\
\text { mothers }\end{array}$ & $\begin{array}{ll}\text { Prevalence } \\
\text { OR } \\
\text { magnitude } \\
\text { estimate } \\
\text { AND } \\
\text { Factors } \\
\text { associated } \\
\text { with } \\
\text { malaria }\end{array}$ & $\begin{array}{l}\text { Quantitative } \\
\text { Studies } \\
\text {. Cohort } \\
\text { study } \\
\text {. } \quad \text { Case } \\
\text { control } \\
\text {. Cross } \\
\text { sectional } \\
\text { studies }\end{array}$ & $\begin{array}{cc}\cdot & \text { English } \\
\text { languages } & \\
\text { Not } \\
\text { limited by } \\
\text { year } \\
\text {. Studies } \\
\text { in } \\
\text { Ethiopia }\end{array}$ \\
\hline $\begin{array}{l}\text { on } \\
\text { a }\end{array}$ & $\begin{array}{l}\text { Children } \\
\text { whose age } \\
\text { group not } \\
\text { well defined }\end{array}$ & ---- & ---- & --- & $\begin{array}{l}\begin{array}{l}\text { Studies } \\
\text { conducted } \\
\text { with: }\end{array} \\
\text { · } \\
\begin{array}{l}\text { Interventio } \\
\text { nal }\end{array} \\
\text { case } \\
\text { report } \\
\text { expert } \\
\text { opinion } \\
\text { qualitative } \\
\text { methods } \\
\text { only }\end{array}$ & $\begin{array}{c}\text { Studies } \\
\text { conducted } \\
\text { in other } \\
\text { than } \\
\text { Ethiopia } \\
\text { Studies } \\
\text { published } \\
\text { with other } \\
\text { languages }\end{array}$ \\
\hline
\end{tabular}

\subsection{PECO search guide}

Population: Under-five children or children 0-59 months old

Exposure: Predictors/determinants of malaria. The determinants are characteristics or exposures that increase the likelihood of malaria among under five children. These may be related to maternal residence, maternal age, educational status, child nutritional status, ITN availability, and distance from malaria breeding sites.

Comparison: The reported reference group for each determinants or associated factors in each study (e.g. malaria in children living in urban area versus children living in rural area, children sleep under ITN versus children not sleeping under ITN beds at night, sex of the children, malnourished child versus well-nourished children, education level of mothers, ... etc).

Outcome: We will include studies those assess prevalence of malaria and its determinants among under five children in Ethiopia. 


\section{Searching strategy}

This meta-analysis will be prepared and presented according to the Preferred Reporting Items for Systematic Reviews and Meta-Analysis [PRISMA, 2009]. We will develop an appropriate and comprehensive search strategy with relevant search terms and pilot test it before the final search. We will search PubMed, MEDLINE, Google Scholar, and Cochrane electronic Databases. We will include articles published from start of indexing until December 30, 2019. We will use Medical Subject Heading (Mesh), keywords, and free text search terms. As the search terms, we will include alternative terms for malaria parasite, and will combine them using Boolean operators. To ensure the comprehensiveness, we will consult an expert librarian. The search strategy for PubMed is supplemented with this protocol (Additional file 2). We will utilize snowballing to screen the references of identified articles for potentially relevant studies. Furthermore, we will contact experts, researchers, and relevant organizations for suggestions on other existing relevant studies. Studies identified by our database searching strategy will be retrieved and managed using Endnote X8 (Thomson Reuters, Philadelphia, PA, USA) software.

Search terms: search (("under-five children" OR "O-59 months old children" OR infants OR neonates)AND ( malaria $O R$ "plasmodium falciparum" $O R$ "plasmodium vivax" $O R$ "mixed malaria" OR "plasmodium species") $A N D$ (Prevalence OR incidence OR magnitude OR burden) AND (determinants OR "risk Factors" OR predictors OR causes OR associated factors") AND (Ethiopia OR Ethio OR Etiopia))

\subsection{Outcome measurement}

This systematic review and meta-analysis will have two main outcomes. The first outcome is estimating the pooled prevalence of malaria among under-five children. Malaria is defined as children with confirmed malaria parasite infection. Pooled prevalence will be calculated by dividing the number of under-five children with malaria to the total number of children who have been included in the study (total sample size) multiplied by 100. The second outcome of the study is to identify maternal and child factors associated with malaria among under-five children. The predictors will be determined in the form of the log odds ratio.

\section{6 selection of studies}

Two authors (AW, and SB) will review the studies, based on inclusion and exclusion criteria. The review will follow three stages. In the first stage, reviewers will assess the titles of the studies identified from the search. In the second stage, abstracts of these selected titles will be included for the final stage of full-text screening. In the third stage, full-text screening, we will screen the full texts selected in the previous stage. If the articles are not open access, we will contact the corresponding author at least for three times. If the authors are not willing to provide the full text, we will exclude that specific article.

In the review, we will only include those studies approved by both authors. The authors will resolve disagreements through discussion or consultation with a third reviewer $(\mathrm{KU})$. We will provide reason for exclusion for all excluded studies. Finally, we will prepare a final list of articles for data extraction

\subsection{Data extraction and management}


Three authors (AW, SB and MD) will independently extracted all necessary data using a standardized data extraction format, which is adapted from the JBI data extraction format for observational studies [24]. We will pretest the data extraction form on three studies of each type, to ensure that it adequately facilitates the collection of all necessary data required for an effective systematic review and meta-analysis. Discrepancies between data extractors will be discussed to reach consensus. If a consensus cannot be reached, the authors will consult a third reviewer $(\mathrm{KU})$. For each included articles, we will record the first author's last name, year of publication, the setting where the study was conducted, study design, study period, sample size, the response rate, the population, outcome definition, comparison groups, and the effect estimate.

\subsection{Quality assessments}

Four authors (AW, SB, KU and MD) will independently conduct quality assessment of included studies, by using the checklist of the JBI appraisal tool for cross-sectional, cohort and case-control studies (Additional file 3). In customising the scale to fit this study, we will took into account the study sampling methods and similarities between the study groups regarding adjustment for confounding factors, the ascertainment of exposure and outcomes, and study design. The abstracting tool will include different questions based on the study designs. The four investigators independently will perform the quality assessment while abstracting the data for the meta-analysis. The quality scores of the four abstractors will be averaged. Finally, the studies with higher scores (>50\%) will be included into meta-analysis.

\subsection{Data synthesis and analysis}

The extracted data will be entered into a Microsoft Excel Database and then imported into STATA version 14.0 (Stata Corp LLC, Texas, USA) software with packages of Meta-analysis for further analysis. The researchers will perform a narrative description of the study population, the studies included, the risk factors identified, and the cause for malaria infection as well as the outcome characteristics. We will use tables and figures to summarize the selected studies and results.

The pooled prevalence of malaria among children under five in Ethiopia will be demonstrated using the random effect model [25]. The Freeman Tuckey variant of the arcsine square root transformation of proportions will be fitted to avoid variance instability when handling proportions close to one [26]. We will assess heterogeneity by using chi-squared test on Cochran's Q statistic with a $5 \%$ level of statistical significance [26] and ${ }^{2}$ statistic test [27], assuming that $\mathrm{I}^{2}$ value of $25 \%, 50 \%$, and $75 \%$ being representative of low, moderate, and high heterogeneity, respectively [27]. If the heterogeneity is significant $\left(\mathrm{I}^{2}>75 \%\right)$, then, we will conduct subgroup analyses and meta-regression to investigate sources of heterogeneity.

Publication bias will be examined by the visual inspection of funnel plots [28] and Egger's test [29]. A p value < 0.10 will be considered indicative of statistically significant publication bias. Thus, if there is evidence of publication bias, we will use Duval and Tweedie's trim-and-fill method [30].

For factors associated with malaria among under-five children; two-by-two tables will be constructed (if possible), the odds ratio with $95 \%$ confidence interval will be calculated. Then, the statistically significance level 
will be declared at a p-value less than 0.05 . However, if the meta-analysis is not possible, we will conduct narrative synthesis.

\subsection{Subgroup and sensitivity analyses}

Sub-group analysis will be performed based on study design, sample size, regions or state, year of publication, quality of studies, and study settings of included studies. by years of publication, study setting, study design, and sample size of the studies. Finally, to conduct sensitivity analysis, we will assess the stability or robustness of the pooled estimates to outliers and the impact of individual studies [30].

\section{Discussion}

This review will provide a detailed summary of the evidence on prevalence and factors of malaria among children in Ethiopia. This review will be the first to synthesis available data on malaria among children. Findings of the review will fill an evidence gap in understanding the burden, risk factors, and causes of malaria in the country. The result from this review will inform health policy planners and researchers up-to-date data on malaria among children and provide direction in which risk factors the policy should focus to reduce burden of malaria among children in the country.

\subsection{Dissemination plan}

The results of this systematic review and meta-analysis will be published in a peer-reviewed journal and presented at national and international research conferences.

\section{Abbreviation}

EDHS Ethiopian Demographic and Health Survey.

JBI Joanna Briggs Institute.

LMICs Low and Middle Income Countries.

MeSH Medical Subject Headings.

PECO Population, Exposure, Comparison, and Outcome.

PRISMA Preferred Reporting Items for Systematic Review and Meta-Analysis.

WHO World Health Organization.

\section{Declarations}

\section{Ethics approval and consent to participate}




\section{Availability of data and materials}

All materials and data related to this article are included in the main document of the manuscript. However, if anyone has interested to have raw data, he/she can contact the corresponding author.

\section{Consent for publication}

Not applicable

\section{Competing interests}

The authors have declared that there are no competing interests.

\section{Funding}

No funding was used.

\section{Acknowledgements}

We would like to thank Samara university librarian and ICT complex staffs for availing an uninterrupted internet connection. We also acknowledge PROSPERO for registration of this protocol.

\section{References}

1. WHO. World malria report. Netherland, Geneva; 2017.

2. WHO. world-malaria-report-2016. Netherland,Geneva; 2016.

3. Kassam R, Sekiwunga R, MacLeod D, Tembe J, Liow E. Patterns of treatment-seeking behaviors among caregivers of febrile young children: a Ugandan multiple case study. BMC Public Health 2016, 16:160.

4. Hajia. Y, Fogartyb. AW, Deressa. W. Prevalence and associated factors of malaria among febrile children in Ethiopia: A cross-sectional health facility-based study. Acta Tropica 2016, 155:63-70.

5. Tadesse. F, Fogarty. AW, Deressa. W. Prevalence and associated risk factors of malaria among adults in East Shewa Zone of Oromia Regional State, Ethiopia: a cross-sectional study. BMC Public Health 2018, 18(25).

6. FMOH: Health and Health Related Indicators. In., vol. 2. Ethiopia: FMOH; 2013: 68.

7. FMOH: Health Sector Development Program IV (2010/11 - 2014/15) FINAL DRAFT. In. Addis Ababa, Ethiopia; 2010.

8. FMOH: Health Sector Transformation Plan (HSTP 2016-2020). In. Addis Ababa, Ethiopia: FMOH; 2015: 184.

9. Nations U: The Millennium Development Goals Report. In.; 2015: 75. 
10. UN.: Transforming our world: the 2030 Agenda for Sustainable Development. sustainabledevelopmentunorg 2015.

11. Nations. U: The Millenium Development Goals 2015 report. In. New york; july 2015.

12. FMoFED.: ASSESSING PROGRESS TOWARDS THE MILLENIUM DEVELOPMENT GOALS: ETHIOPIA MDGs REPORT 2012. In. Addis Ababa, Ethiopia; 2012: 42.

13. Jima. D, Wondabeku. M, Alemu. A, Teferra. A, Awel. N, Deressa. W, Adissie. A, Tadesse. Z, Gebre. T, Mosher. AW et al: Analysis of malaria surveillance data in Ethiopia: what can be learned from the Integrated Disease Surveillance and Response System? Malaria Journal 2012, 11(330).

14. Ashton RA, Kefyalew T, Tesfaye G, Pullan RL, Yadeta D, Reithinger R, Kolaczinski JH, Brooker S: Schoolbased surveys of malaria in Oromia Regional State, Ethiopia: a rapid survey method for malaria in low transmission settings. Malar J 2011, 10:25.

15. Ayalew. S: The Prevalence of Malaria and the Associated Risk Factors in Jiga area, Northwest Ethiopia. In. Addis Ababa: AAU; 2014.

16. Debo. GW, Kassa. DH: Prevalence of malaria and associated factors in Benna Tsemay district of pastoralist community, Southern Ethiopia. Tropical Diseases, Travel Medicine and Vaccines 2016, 2(16).

17. Molla. E, Ayele. B: Prevalence of Malaria and Associated Factors in Dilla Town and the Surrounding Rural Areas, Gedeo Zone, Southern Ethiopia. J Bacteriol Parasitol 2015, 6(5).

18. Roberts D, Matthews G: Risk factors of malaria in children under the age of five years old in Uganda. Malar J 2016, 15:246.

19. Tassew. A, Hopkins. R, Deressa. W: Factors influencing the ownership and utilization of long-lasting insecticidal nets for malaria prevention in Ethiopia. Malaria Journal 2017, 16(262).

20. CSA.: ETHIOPIA Demographic and Health Survey 2016. In. Addis Ababa, Ethiopia; 2017.

21. ARHB.: Afar Regional Health Bureau 2017 malaria report. In. Semera, Ethiopia; 2017.

22. Moher D, Liberati A, Tetzlaff J, Altman DG: Preferred reporting items for systematic reviews and metaanalyses: the PRISMA statement. Bmj 2009, 339:b2535.

23. Shamseer L, Moher D, Clarke M, Ghersi D, Liberati A, Petticrew M, Shekelle P, Stewart LA: Preferred reporting items for systematic review and meta-analysis protocols (PRISMA-P) 2015: elaboration and explanation. BMJ : British Medical Journal 2015, 349:g7647.

24. Institute JB: Joanna Briggs Institute Reviewers Manual 2014 Edition. In., 2014 edn. Australia: the University of Adelaide; 2014.

25. Berkey CS, Hoaglin DC, Mosteller F, Colditz GA: A random-effects regression model for meta-analysis. Stat Med 1995, 14(4):395-411.

26. NN. V, A. M, A. M: Metaprop: a Stata command to perform meta-analysis of binomial data. Archives ofPublic Health 2014, 72(39).

27. Higgins JP, Thompson SG: Quantifying heterogeneity in a meta-analysis. Stat Med 2002, 21(11):1539-1558.

28. Liu JL: The role of the funnel plot in detecting publication and related biases in meta-analysis. Evidencebased dentistry 2011, 12(4):121-122.

29. Egger M, Davey Smith G, Schneider M, Minder C: Bias in meta-analysis detected by a simple, graphical test. Bmj 1997, 315(7109):629-634. 
30. Duval S, Tweedie R: Trim and fill. A simple funnel-plot-based method of testing and adjusting for publication bias in meta-analysis. Biometrics 2000, 56(2):455-463.

\section{Additional Files}

\subsection{Additional file 1: PRISMA-P 2015 checklist}

PRISMA-P (Preferred Reporting Items for Systematic review and Meta-Analysis Protocols) 2015 checklist: recommended items to address in a systematic review protocol 
Section and Item

Checklist item

Page

topic No

number

ADMINISTRATIVE INFORMATION

Title:

1a Identify the report as a protocol of a systematic review

1

Identification

Update $\quad 1 \mathrm{~b}$ If the protocol is for an update of a previous systematic review, identify as such

Not applicable

Registration 2 If registered, provide the name of the registry (such as PROSPERO) and registration number PROSPERO- CRD42020157886

Authors:

Contact 3a Provide name, institutional affiliation, e-mail address of all protocol authors; provide physical mailing

5 address of corresponding author

3b Describe contributions of protocol authors and identify the guarantor of the review

Contributions

Amendments $\quad 4$ If the protocol represents an amendment of a previously completed or published protocol, identify as such and list changes; otherwise, state plan for documenting important protocol amendments

Not

Applicable

Support:

Sources $5 a$ Indicate sources of financial or other support for the review

Not

Sponsor 5b Provide name for the review funder and/or sponsor

Applicable

Not

Role of 5c Describe roles of funder(s), sponsor(s), and/or institution(s), if any, in developing the protocol

Applicable

Not

sponsor or

Applicable

funder

\section{INTRODUCTION}

\begin{tabular}{lllc}
\hline Rationale & 6 & Describe the rationale for the review in the context of what is already known & 3 \\
\hline Objectives & 7 & $\begin{array}{l}\text { Provide an explicit statement of the question(s) the review will address with reference to participants, } \\
\text { interventions, comparators, and outcomes (PICO/PECO) }\end{array}$ & 3 \& 6 \\
\hline
\end{tabular}

METHODS

Eligibility criteria 8 Specify the study characteristics (such as PICO, study design, setting, time frame) and report characteristics (such as years considered, language, publication status) to be used as criteria for eligibility for the review

Information 9 Describe all intended information sources (such as electronic databases, contact with study authors, trial registers or other grey literature sources) with planned dates of coverage

sources

Search strategy 10 Present draft of search strategy to be used for at least one electronic database, including planned limits, such that it could be repeated

\section{Additional file 2}

\section{Study records:}

Data management

Selection

11a Describe the mechanism(s) that will be used to manage records and data throughout the review

process

Data

collection

process

Data items

11b State the process that will be used for selecting studies (such as two independent reviewers) through each phase of the review (that is, screening, eligibility and inclusion in meta-analysis)

11c Describe planned method of extracting data from reports (such as piloting forms, done independently, in duplicate), any processes for obtaining and confirming data from investigators

12 List and define all variables for which data will be sought (such as PICO items, funding sources), any preplanned data assumptions and simplifications

Outcomes and 13 List and define all outcomes for which data will be sought, including prioritization of main and additional prioritization outcomes, with rationale

Risk of bias in individual

14 Describe anticipated methods for assessing risk of bias of individual studies, including whether this will

studies be done at the outcome or study level, or both; state how this information will be used in data synthesis

Data synthesis 15a Describe criteria under which study data will be quantitatively synthesized

$15 \mathrm{~b}$ If data are appropriate for quantitative synthesis, describe planned summary measures, methods of handling data and methods of combining data from studies, including any planned exploration of 
consistency (such as $I^{2}$, Kendall's $\tau$ )

15c Describe any proposed additional analyses (such as sensitivity or subgroup analyses, meta-regression) 9

$15 \mathrm{~d}$ If quantitative synthesis is not appropriate, describe the type of summary planned

16 Specify any planned assessment of meta-bias(es) (such as publication bias across studies, selective reporting within studies)

Meta-bias(es)

Confidence in cumulative

evidence

17 Describe how the strength of the body of evidence will be assessed (such as GRADE)

*It is strongly recommended that this checklist be read in conjunction with the PRISMA-P Explanation and Elaboration (cite when available) for important clarification on the items. Amendments to a review protocol should be tracked and dated. The copyright for PRISMA-P (including checklist) is held by the PRISMA-P Group and is distributed under a Creative Commons Attribution Licence 4.0.

From: Shamseer L, Moher D, Clarke M, Ghersi D, Liberati A, Petticrew M, Shekelle P, Stewart L, PRISMA-P Group. Preferred reporting items for systematic review and meta-analysis protocols (PRISMA-P) 2015: elaboration and explanation. BMJ. 2015 Jan 2; 349 (jan02 1):g7647.

\subsection{Additional file 2: PubMed search string}




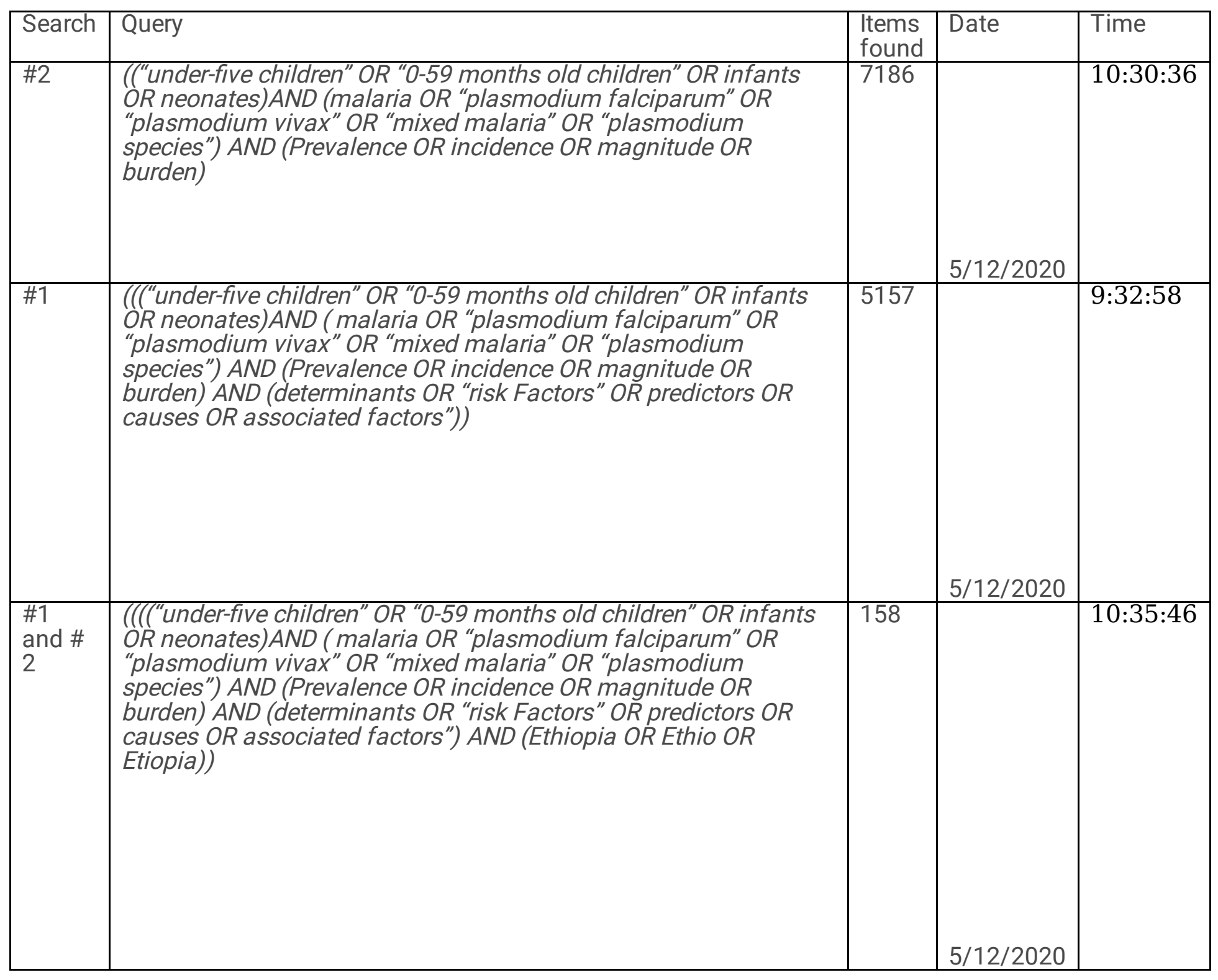

\subsection{Additional 3: JBI quality appraisal checklist}

JBI quality assessment tools available: https://joannabriggs.org/ebp/critical_appraisal_tools 